\title{
La amazonía colombiana como sujeto de derechos: caracterización del conflicto ambiental que llevó a su reconocimiento
}

\author{
The colombian amazon as a subject of rights: recognition and characterization of \\ the environmental conflict \\ *IVÁN VARgas-Chaves \\ ${ }^{*}$ Mauricio LunA-Galván \\ *** Katy TORRES-PÉREZ
}

* Doctor en Derecho. Profesor titular de la Universidad La Gran Colombia. Correo: ivan.vargas@ugc.edu.co; ORCID: 0000-0001-6597-2335

**Máster en Estudios Globales. Profesor vinculado de la Universidad La Gran Colombia. Correo: mauricio.luna@ugc.edu.co; ORCID: 0000-0002-4594-4940

***Asistente de investigación. Universidad Tecnológica de Bolívar. ORCID: 0000-0002-2209-877X

Fecha de recepción: 15 agosto de 2019 Fecha de aprobación: 4 de diciembre de 2019

Para citar este artículo / To reference this article Vargas-Chaves, I. Luna-Galván, M. Torres-Pérez, K (2019) La amazonía colombiana como sujeto de derechos: caracterización del conflicto ambiental que llevó a su reconocimiento. Inciso, 21(2) ; 146-160.

Resumen

DOI: http://dx.doi.org/10.18634/incj.21v.2i.990

A través de la aplicación de un instrumento metodológico de caracterización de conflictos ambientales, en el presente artículo se lleva a cabo un ejercicio descriptivo de los antecedentes del conflicto que llevó al reconocimiento de la Amazonía colombiana como sujeto de derechos por parte de la Corte Suprema de Justicia. Adicional a lo anterior, y con el propósito de estructurar este estudio de caso se relacionan las posturas de los actores principales y secundarios, además de los aspectos sociales, económicos y ambientales identificados en la acción de tutela mediante la aplicación de un instrumento de caracterización de conflictos en el que se examinan los principales actores y factores sociales, gubernamentales, económicos y geográficos que desencadenaron la problemática ambiental y que permiten desde diferentes ópticas su comprensión. Por último, se presentan a manera de conclusiones las consideraciones que desde el biocentrismo le permitieron a la Corte fundamentar esta novedosa visión. 
Palabras clave: Amazonía colombiana, biocentrismo, conflictos ambientales, derechos de la naturaleza.

\begin{abstract}
In this paper, the authors apply an instrument to characterize environmental conflicts in the case-study of the Colombian Amazon, which was recognized as a subject of rights by the Supreme Court of Justice. To achieve this objective, the paper describes the background of the conflict, the positions of the main and secondary actors, as well as the social, economic and environmental impacts identified by the main actors by applying a conflict characterization instrument. This instrument examines the main actors and social, governmental, economic and geographical factors that caused the socio-environmental problems to generate a space for analysis from different perspectives. Finally, the authors identify the considerations of the Court to support this new vision from biocentrism.
\end{abstract}

Keywords: Biocentrism; Colombian Amazon; Environmental conflicts; Rights of nature

\title{
Introducción
}

La Amazonía colombiana que hace parte de ese gran ecosistema conocido como 'el pulmón del mundo' es un territorio que se destaca por sus recursos naturales, por una biodiversidad compuesta por multiplicidad de especies animales, una gran variedad de especies forestales y especies endémicos (Armenteras, Rudas, Rodríguez, Sua y Romero 2006), además ser el 4,9\% del área continental mundial con la cuenca más grande del mundo y el $20 \%$ de agua dulce del planeta (Veyrunes, 2008).

Esta vasta e importante región ha experimentado de manera vertiginosa en las últimas décadas un proceso de deterioro debido a factores como la deforestación y la contaminación; consecuencia de la preeminencia de una economía de rentabilidad a corto plazo, lo cual ha desencadenado alteraciones en los ciclos climáticos y de precipitaciones (Programa de las Naciones Unidas para el Medio Ambiente, PNUMA, 2009).

El conflicto ambiental que en el presente artículo se analizará, encuentra su punto de partida en una acción de tutela instaurada por 25 ciudadanos frente la omisión del Estado colombiano ante las alertas de deforestación que se emitieron en el primer trimestre de 2017 como consecuencia del acaparamiento de tierras, cultivos ilícitos, extracción minera ilegal, infraestructura, cultivos agroindustriales y extracción ilegal de madera, así como de los reportes de emisión de gases efecto invernadero, afectación del suelo para absorber líquidos así como de un potencial riesgo de extinción de especies endémicas (Instituto de Hidrología, Meteorología y Estudios Ambientales, Ideam, 2017).

El enfoque biocentrista ha buscado poner de relieve el rol que ha adquirido el activismo judicial para la solución de conflictos ambientales, tal como ocurre en el caso que aquí se estudia. Para ello, se lleva a cabo de un lado una identificación de este conflicto a través de un instrumento metodológico de caracterización de conflictos ambientales propuesto por Rodríguez (2014), y del otro, se estudian las consideraciones que llevaron a la Corte Suprema de Justicia a otorgar el estatus de sujeto de derechos a la Amazonía colombiana. 
Por lo demás, con este instrumento se busca identificar cada aspecto clave del conflicto con el fin de comprender el curso del mismo. A través de una serie de indicadores validados en la metodología de caracterización se estudian cuestiones que dan alcance no solo a la identificación de factores, sino a la definición misma del conflicto a partir de actores, hechos, ubicación, aspectos sociales y descripción de los recursos utilizados para solucionarlo. A continuación, se presentan los resultados tras aplicar el instrumento, por último, unas reflexiones finales a manera de conclusiones.

\section{Planteamiento de la problemática}

A partir del momento en el que el ser humano empezó a adquirir conciencia sobre el impacto de las actividades industriales en el ambiente, muchos e importantes avances se han alcanzado. Desde el cambio en sus hábitos de consumo hasta el advenimiento de tendencias como la ecología política, la responsabilidad social de las empresas o la inteligencia verde, se proyecta una tendencia que influye en las decisiones y en el actuar de nuestra especie para mejorar las condiciones de vida en este planeta.

El biocentrismo visto desde el enfoque del reconocimiento de la naturaleza como sujeto de derechos ha sido un aporte crucial que desde el ámbito jurídico y político, se ha dado en este sentido. En América Latina se materializó, por ejemplo, con las constituciones políticas de Ecuador en 2008 y de Bolivia en 2009.

En el primer caso, en su preámbulo se apeló a este concepto al considerar que el ser humano es y forma parte de la naturaleza estando en una misma categoría cuando expresa:

[...] la Pacha Mama, de la que somos parte y que es vital para nuestra existencia (...) donde se reproduce y realiza la vida, tiene derecho a que se respete integralmente su existencia y el mantenimiento y regeneración de sus ciclos vitales, estructura, funciones y procesos evolutivos (Constitución Política de la República de Ecuador, artículo 71).

Por su parte la constitución boliviana pese a no reconocer a la naturaleza como sujeto de derechos de manera directa, sí presenta una marcada tendencia biocéntrica. En el preámbulo se establece que: "Cumpliendo con el mandato de nuestros pueblos, con la fortaleza de nuestra Pachamama y gracias a Dios, refundamos Bolivia", refiriéndose en el capítulo $V$ sobre los derechos de la naturaleza que:

Las personas tienen derecho a un medio ambiente saludable, protegido y equilibrado. El ejercicio de este derecho debe permitir a los individuos y colectividades de las presentes y futuras generaciones, además de otros seres vivos, desarrollarse de manera normal y permanente. (Constitución Política de 2009 del Estado Plurinacional de Bolivia, artículo 33).

La relevancia de estos dos textos constitucionales viene dada por su carácter integrador con las cosmovisiones de los pueblos indígenas que habitan el territorio. En el caso de la Constitución Política boliviana se establece en su artículo 73 que la naturaleza, o Pacha Mama, tendrá derecho a su restauración; entendida no como la indemnización a los pueblos y comunidades que vean afectados sus derechos por los impactos ambientales, sino como el proceso de recuperación de los ecosistemas (Gudynas, 2009). 
A su vez, el ordenamiento jurídico boliviano contempla, por un lado, instituciones como la Defensoría y la Autoridad Nacional de la Madre tierra, por el otro, las denominadas leyes de la Madre Tierra - Ley 071 de 2010 y la Ley 300 de 2012- en las que se da alcance al reconocimiento de los derechos a la naturaleza, asimilados como un sistema compuesto por seres vivos, indivisibles, interdependientes, interrelacionados y complementarios, de cara a la preservación de ciclos productores y generadores de vida como el agua o el aire.

No en vano en el biocentrismo se concibe la transición de una visión antropocéntrica a una en la cual es el ser humano, quien se convierte en un elemento perteneciente a ese 'ser dinámico' que es la naturaleza, coexistiendo con ella al igual que los demás seres que la habitan, componen y a todos los fenómenos que en ella tienen lugar.

En el caso de Colombia, pese a que su Constitución Política ha sido denominada por la doctrina como la 'Constitución Ecológica' dada la gran importancia que para la misma revista el ambiente como un interés jurídico tutelado clave dentro de los derechos colectivos (Rodríguez y Páez, 2012), el reconocimiento de la naturaleza como sujeto de derechos no se ha dado aún en el ámbito legislativo, como sí en el jurisprudencial a través de la sentencia T-622 de 2016 de la Corte Constitucional que reconoció al río Atrato como sujeto de derechos.

De hecho la importancia de esta decisión, además del hecho de ser el primer antecedente de este reconocimiento, radica en el análisis realizado por este alto tribunal al contemplar integralmente la protección de los derechos a un ambiente sano, salud, agua y soberanía alimentaria desde un único enfoque, en un caso de actividad minera ilegal desarrollada en el río Atrato.

Para entonces, la Corte Constitucional reconoció que el río Atrato es un sujeto de derechos con especial protección para evitar el continuo deterioro ocasionado por la maquinaria utilizada y el vertimiento de sustancias tóxicas en el agua como el mercurio y cianuro. Lo relevante del caso es que a partir de esta decisión en dos sentencias más -incluida la ST4360 de 5 de abril de 2018 que en este artículo abordaremos a través de la caracterización del conflicto ambiental- se dio alcance a este postulado; siendo la otra sentencia la que profirió el Tribunal Administrativo de Boyacá que le otorgó el reconocimiento al Páramo de Pisba como un sujeto de derechos.

\section{Materiales y métodos}

Para el análisis e identificación de los conflictos ambientales, Rodríguez (2014) elaboró un instrumento metodológico que recopila datos clave para caracterizar un conflicto de este tipo. Con el fin de obtener la información necesaria para implementarlo se hace preciso el detallado diligenciamiento de un cuestionario a través de respuestas soportadas en análisis de documentación, etnografía, cartografía social u otras técnicas para recoger información que se va a analizar. Esto le permite al investigador identificar y validar desde los hechos que originaron el conflicto hasta las vías adoptadas para la resolución del mismo.

Así, en primer lugar, el instrumento contempla (i.) la identificación y definición del conflicto; (ii.) su ubicación y; (iii.) una identificación de los aspectos sociales. En segundo lugar, se estudian los (iv.) procesos y (v.) aspectos sustanciales del problema, para finalizar con (vi.) descripción detallada de los recursos para resolver el conflicto y la manera en que estos se implementaron. 
Con la finalidad de comprender plenamente el curso de cada uno de los aspectos a incluir en el instrumento, se responden preguntas-problema que dan cuenta del alcance metodológico de esta caracterización. Así, por ejemplo, para la identificación y definición del conflicto las preguntas a responder fueron ¿Cuál es la situación planteada que genera conflicto? ¿Qué instituciones están o pueden vincularse con el conflicto? ¿Cuáles son los hechos que ocasionaron el conflicto? (Rodríguez, 2014).

En últimas, lo que se busca a través del instrumento es llegar a cuestiones clave del conflicto con la finalidad de dimensionar el estado de vulnerabilidad en el que se encuentran los stakeholders implicados -entre estos las comunidades o el ambiente- y determinar el curso que este tomó.

\section{Aplicación del instrumento de caracterización de conflictos ambientales}

\section{Identificación y definición del conflicto}

La vía escogida para solucionar el conflicto ambiental que aquí se examina se dio con una acción de tutela interpuesta por el Centro de Estudios Dejusticia en conjunto con un grupo de jóvenes provenientes de distintas ciudades del país, todas ellas con riesgos potenciales por los efectos del cambio climático. En la acción se le solicitó al Estado colombiano tutelar sus derechos a la salud; a un ambiente sano, al agua, alimentación y a una vida digna. Ello, en tanto se han dado situaciones que evidencian una negligencia en su actuar y en el incumplimiento de sus deberes como garante de estos derechos.

Cabe resaltar que con anterioridad, el gobierno colombiano había adquirido el compromiso (Cf. Acuerdo de París, 2015; Ley 1753 de 2015) de implementar medidas que llevaran a la reducción de la deforestación en la Amazonía hasta llegar a cero, así como a desarrollar una estrategia para disminuir los gases de efecto invernadero, principales agentes del cambio climático en Colombia.

Con todo, en instrumentos de medición de políticas públicas como la Estrategia Integral de Control a la Deforestación y Gestión de los Bosques en Colombia del Ministerio de Ambiente y Desarrollo Sostenible se evidencia que en 2016 la Amazonía había seguía perdiendo terreno; en total 178.597 hectáreas. La deforestación en Colombia en comparación con el año inmediatamente anterior no solo aumento en un 44\%, sino que de esa cifra 70.074 hectáreas corresponden a la Amazonía (Rodríguez et al., 2018).

De igual forma, para el 2017 la Amazonía de Colombia fue la región con mayor nivel de Alertas Tempranas de Deforestación (AT-D) alcanzando un 66,2\% del total, de acuerdo con el Boletín emitido por el Ministerio de Ambiente y Desarrollo Sostenible y el Ideam.

Otro aspecto relevante es que las corporaciones autónomas Corpoamazonía y Cormacarena, entidades públicas encargadas de administrar los recursos naturales y para propender por el desarrollo sostenible de sus ecosistemas, constituyeron en 2016 reservas presupuestales en cuantías que superan las dispuestas por el Decreto 1957 de 2007; reduciendo así la disponibilidad presupuestal para combatir la deforestación según informe sobre los Recursos Naturales y del Ambiente de la Contraloría General de la Nación en el 2017 (Ministerio de Ambiente y Desarrollo Sostenible, 2017). 
En definitiva, la deforestación estaba ocasionando un daño irreparable, consecuencia de problemas sin resolver como la masificación de cultivos de uso ilícito, acaparamiento de tierras en el marco del conflicto armado, extracciones mineras ilegales y tala ilícita de árboles, infraestructura y cultivos agroindustriales (Rodríguez et al., 2018).

\section{Ubicación}

El caso en estudio, se desarrolla en los municipios de San Vicente del Caguán, Cartagena del Chairá, San José del Guaviare, Calamar, La Macarena, Puerto Leguízamo, Solano, Uribe, El Retorno, Puerto Guzmán, Puerto Rico, Miraflores, Florencia y Vistahermosa pertenecientes a los departamentos con mayor extensión territorial de selva amazónica, Amazonas, Caquetá, Guaviare y Putumayo.

Pese a que la problemática pareciera ser de tipo regional, no es algo que afecte únicamente a estos entes territoriales. Se torna de carácter nacional en tanto que en la zona se encuentran cuatro parques nacionales naturales de Colombia, a saber, el Parque de la Sierra de la Macarena, Parque Nacional Natural Nukak, Tinigua y La Paya, los cuales poseen una amplia variedad de flora y fauna entre aves, peces y mamíferos. (Parques Nacionales Naturales, 2018). Estos ocuparon los lugares 3, 5, 6 y 9 respectivamente en la clasificación de parques con mayor concentración de AT-D en el 2017 (Ideam, 2017).

No obstante, se debe tener en cuenta que la Amazonía no reviste importancia solo para los países que la integran. Su relevancia va más allá, siendo de índole global debido a la riqueza natural, social y cultural, teniendo este ecosistema el área de bosque tropical más extensa, así como la fuente hídrica más grande del planeta con el Río Amazonas que es el más largo, caudaloso, ancho y profundo en la tierra (PNUMA, 2009).

Por tal motivo, no podría ser de otra manera, tal como lo expresó la Corte Suprema de Justicia, la conservación de la Amazonía no es solo competencia nacional sino global al ser el principal eje ambiental y ecosistémico del planeta.

Ello, máxime si se tiene en cuenta que la inoperancia e inacción de las autoridades colombianas en la protección del hábitat más biodiverso del mundo afecta a países vecinos como Brasil, Bolivia, Perú, Guyana, Venezuela, Surinam y la Guyana Francesa, quiénes también poseen territorio amazónico (Rodríguez et al., 2018).

Las consecuencias de esta degradación ambiental sin control, pueden ser a corto, mediano y largo plazo, ocasionando un perjuicio inminente para la vida digna de generaciones presentes y futuras. Los efectos de este problema ambiental, tal como fueron descritas por los actores en la acción de tutela son de diversa índole.

En primer lugar, se encuentra una proyección negativa en toda la región andina al alterarse el ciclo del agua (Urrutia y Vuille, 2009). Al respecto, los bosques amazónicos absorben el agua del suelo y luego, por evapotranspiración restituyen el agua a la atmósfera contribuyendo a las precipitaciones y lluvias que constituyen el $50 \%$ de las que caen en esa zona, las cuáles terminan en escorrentía alimentando el caudal del río Amazonas (Mariné, 2007; Bunyard y Herrera, 2012).

Producto de la deforestación se disminuyen los principales agentes de producción del ciclo del agua disminuyendo las precipitaciones y generando sequías, además de afectar a las poblaciones 
que se abastecen del río para el suministro de agua, pesca, riego de cultivos e incluso recreación (Rodríguez et al., 2018).

En segundo lugar, debido a la deforestación las raíces de los árboles ven interrumpida su labor de captación del agua del suelo derivando en el fenómeno conocido como saturación de la capa freática -capa de agua que hay en el subsuelo- y las consecuentes inundaciones (Pinzón-Pinto, 2010).

Por otro lado, la deforestación contribuye a romper la conectividad existente entre el ecosistema amazónico y la cordillera andina, debido a que el agua evotranspirada de los bosques sube a la atmósfera -gracias a los vientos alisios-convirtiéndose en agua condensada que cae en forma de lluvia al hábitat andino, nutriendo el agua que absorben los páramos y corrientes de agua que llegan a los cultivos, y abastecen a medianas y grandes ciudades (Bunyard y Herrera, 2012), pudiendo afectar incluso sectores como el energético, industrial y agrícola (Pereira, 2001).

Finalmente, con la deforestación y quema de bosques se produce la emisión de dióxido de carbono que antes estaba contenida en los árboles y con su tala se liberan en el aire, en el ambiente, y producen un cambio climático al ser el principal gas de efecto invernadero. Como resultado de ello se tiene que la temperatura en Colombia para el período comprendido entre el 2041 y 2071 aumente entre $1,6^{\circ}$ a $2,14^{\circ}$ (Rodríguez et al., 2018). De igual forma, el calentamiento del aire genera un ambiente más húmedo, lo que contribuye a la propagación de enfermedades como la malaria, el dengue y el zika (Padilla, Lizarazo, Murillo, Mendigaña, Pachón y Vera,, 2017).

\section{Aspectos sociales [actores]}

Al identificar los actores participantes en el desarrollo del conflicto, se encuentra como el primero de estos a la sociedad misma, un actor colectivo, representado por el conjunto de 25 jóvenes, niños y adolescentes -acompañados por Dejusticia- que interpusieron una acción de tutela dada la creciente deforestación en la Amazonía colombiana, y en tanto que ello vulneraba su derecho a gozar de un ambiente sano, en los términos del artículo 79 de la Constitución Política, entre otros derechos fundamentales como la vida digna, la salud, el agua y la alimentación.

Como actores secundarios - en calidad de accionados-se relacionan las autoridades ambientales, entre estas (i.) la Unidad Administrativa de Parques Nacionales Naturales como autoridad del orden nacional, encargada de administrar el Sistema de Parques Nacionales Naturales y el Sistema Nacional de Áreas Protegidas de Colombia. Están además (ii.) la Corporación para el Desarrollo Sostenible del Norte y el Oriente Amazónico -CDA-; (iii.) la Corporación para el Desarrollo Sostenible del Sur de la Amazonía -Corpoamazonía-; y (iv.) la Corporación para el Desarrollo Sostenible de la Macarena - Cormacarena-.

Todas estas en calidad de entidades territoriales, parte del Sistema Nacional Ambiental, que tienen dentro de su función administrar en el espacio de su jurisdicción los recursos naturales renovables en búsqueda de estrategias dinámicas en pro del desarrollo sostenible. (Ministerio de Ambiente y Desarrollo Sostenible, 2018).

Para el caso de la Corpoamazonía, la Ley 99 de 1993 en su artículo 35 establece específicamente que es su función: "Proteger el medio ambiente del sur de la Amazonía colombiana como área 
especial de reserva ecológica de Colombia, de interés mundial y como recipiente singular de la mega-biodiversidad del trópico húmedo", además, de concederle funciones de asesoramiento a los municipios para la creación de normativas que protejan el medio natural.

En lo referente a Cormacarena esta misma norma le asignó funciones específicas de cuidado y promoción de: "La conservación y el aprovechamiento sostenible de los recursos naturales renovables y del medio ambiente del área de Manejo Especial La Macarena" (Ley 99 de 1993, artículo 38).

Lo relevante del caso es que en la jurisdicción de estas dos entidades se reportaron niveles de deforestación para el período del último trimestre de 2017 . Con un $24,47 \%$ y $14,74 \%$ respectivamente, de un total de 47, 23\% de AT-D (Rodríguez et al., 2018).

Respecto a los agentes administrativos, son actores secundarios el poder ejecutivo en cabeza de la Presidencia de la República, y los ministerios de Ambiente y Desarrollo Sostenible de un lado, y, del otro el de Agricultura y Desarrollo Rural.

De manera especial, vale la pena destacar que el Ministerio de Ambiente y Desarrollo Sostenible tiene la obligación de actuar frente a la omisión de las corporaciones autónomas regionales frente al deterioro ambiental, ejerciendo labores de evaluación, control preventivo actual o posterior e incluso de órdenes de suspensión de actividades de explotación, exploración entre otras con el fin de proteger los recursos naturales renovables (Ley 99 de 1993, artículo 5).

Otros actores secundarios son los de orden regional, entre estos los departamentos y municipios amazónicos que componen las subregiones de la Amazonía colombiana, cuya obligación es la de incluir en sus planes de ordenamiento territorial políticas para la conservación del ecosistema amazónico.

\section{Proceso y postura de los actores}

Los actores principales presentan una acción de tutela bajo las consideraciones de que las omisiones de las autoridades administrativas y en materia ambiental -para la protección de la región amazónica y los ecosistemas que en ella subsisten- amenazan su derecho a gozar de un ambiente sano; poniendo además en riesgo sus derechos a la vida, agua, alimentación y salud. Ello, puesto que dicha omisión ha favorecido el incremento en esta zona del país, situación que ha provocado la liberación de gases de efecto invernadero -entre los que se encuentra el $\mathrm{CO}_{2}$ - los cuales son los principales agentes del cambio climático.

La legitimación de su postura como actores principales yace en el hecho que siendo precisamente ellos, como jóvenes pertenecientes a la generación futura, quienes se verán obligados a enfrentar las consecuencias del cambio climático (Salamanca et al., 2018) tienen la posibilidad de acceder al aparato jurisdiccional con el fin de solicitar el amparo de sus derechos.

Al respecto, al tener un promedio de vida de 78 años, desarrollarán su vida adulta entre los años 2040-2070 y parte de su vejez desde el 2070 en adelante, periodo dentro de los que según reportes del Ideam se espera un aumento promedio de la temperatura de entre 1,6 y 2,14 grados respectivamente; generando sequías en algunas regiones del territorio y extremas 
precipitaciones en otras (Rodríguez et al., 2018).

Por tal razón, según se lee en el documento de la acción, los actores en representación de las generaciones venideras verán mermado su proyecto de vida. En lo referente al derecho al agua, afirma el grupo de 25 jóvenes que se vulnera la accesibilidad, pues con los gases de efecto invernadero que ocasionan el cambio climático se evidenciará una disminución en las precipitaciones en el $27 \%$ del territorio nacional (Rodríguez et al., 2018).

También se proyecta una potencial amenaza a su acceso en regiones con dificultades de comunicación dado que las inundaciones en algunos sectores podrían dejar incomunicadas a ciertas poblaciones impidiendo el transporte y el suministro de agua potable. Finalmente, se vería perjudicado el ecosistema que produce la lluvia, debido a que el 50\% del agua que cae en la Amazonía es agua de lluvia reciclada, la cual está desapareciendo con el fenómeno de la deforestación (Bunyard y Herrera, 2012).

En cuanto al derecho a la salud, con la deforestación se producen varios cambios en las precipitaciones, disminuyéndose en algunos casos, lo que genera que las personas almacenen el recurso hídrico sin condiciones sanitarias, tal como se está evidenciando en algunas poblaciones de la Amazonía brasileña (Bernardes y Riso, 2014). Por otro lado, al aumentarse las precipitaciones, se darían 'encharcamientos' que podrían ser el escenario perfecto para la proliferación de vectores, por ende, de enfermedades (Arria, Rodríguez-Morales y Franco-Paredes, 2005).

En cuanto a la soberanía alimentaria, con la deforestación, los gases de efecto invernadero y su derivación en el cambio de las temperaturas, se afectarían las dinámicas de los ecosistemas del Amazonas, pudiéndose generar el desabastecimiento en poblaciones de toda la región tropical andina (Urrutia y Vuille, 2009; Viviroli et al., 2011).

Bajo estas premisas, los actores principales del conflicto deciden acudir utilizando el mencionado mecanismo judicial para solicitar la salvaguarda de sus derechos, y los de la Amazonía colombiana ante el Tribunal Superior del Distrito Judicial de Bogotá, quien pese a reconocer gran parte de la problemática antes descrita, desestima las pretensiones y niega el amparo de la solicitud de tutela del derecho a gozar de un ambiente sano en conexidad con los derechos fundamentales.

Esta fue la decisión que los actores principales del conflicto decidieron impugnar ante la Sala Civil de la Corte Suprema de Justicia, quien otorgaría el amparo constitucional al derecho al ambiente y los derechos fundamentales de los accionantes a la vida, agua, alimentación y salud.

Ello, al considerar los principios rectores en materia ambiental, siendo el primero de ellos el principio de precaución, contenido en la Declaración de Río sobre Medio Ambiente y Desarrollo de 1992, según el cual, los Estados deben tomar medidas tendientes a la protección del medio ambiente cuando exista peligro de daño irreversible, aun cuando la certeza científica sobre este no sea absoluta, pues esta no será razón para no tomar acciones que propugnen por una eficaz protección de la naturaleza y evitar así la degradación del medio ambiente (Cameron y Abouchar, 1991).

Durante el proceso, las autoridades accionadas manifestaron una postura evasiva de sus responsabilidades como guardianes de la naturaleza. Los argumentos esgrimidos fueron, en síntesis, los siguientes: 
(i.) La presidencia pidió ser desvinculada dentro del proceso por considerar que no vulnera los derechos fundamentales de los menores accionantes. (ii.) El Ministerio de Agricultura y Desarrollo Rural afirmó carecer de competencia para actuar dentro del presente asunto, el Ministerio de Ambiente y Desarrollo Sostenible se limitó a explicar cuáles eran las estrategias nacionales diseñadas para hacer frente a la deforestación y las gestiones para el cambio climático.

A su vez, (iii.) Parques Nacionales Naturales expresó adelantar lo mejor posible su labor con los medios que dispone. Por su parte, como intervinientes de orden departamental (iv.) la Gobernación de Guaviare y la Gobernación de Caquetá manifestaron haber adelantado gestiones tendientes a la protección de la Amazonía colombiana, mientras que la Gobernación del Putumayo se adhirió al sentido del fallo del Tribunal. La Alcaldía de San Vicente del Caguán expuso no haber incurrido en quebranto alguno, por último, la Alcaldía de Solano y Corpoamazonía adujeron trabajar y realizar todo lo necesario para la protección de la región amazónica.

De lo anterior se observa que, en su mayoría, las autoridades competentes tanto de orden nacional como departamental y municipal, adoptaron una postura contraria a las peticiones de los accionantes. La pregunta que inevitablemente surge al respecto es si en la visión política-administrativa de los gobiernos colombianos y las clases dirigentes, ¿existe realmente una preocupación por incluir dentro de sus planes y listas, la defensa de la región amazónica y de toda su biodiversidad?, o por el contrario, ¿en sus objetivos solo está el aprovechamiento económico de las actividades extractivas e industriales sin prever un desarrollo sostenible?

\section{Descripción de los recursos y la manera cómo se resolvió el conflicto}

Dentro del proceso como se mostró, se discutió la procedibilidad de la acción de tutela como mecanismo idóneo para la protección del derecho a gozar de un ambiente sano y conexamente a otros derechos de orden fundamental. En este sentido el artículo 88 de la Constitución Política establece que para la protección de los derechos colectivos -entre estos el derecho a gozar de un ambiente sano-, los afectados pueden acudir en grupo ante el aparato jurisdiccional a través de la acción popular, en los términos de la Ley 472 de 1998.

Esta fue la línea argumentativa planteada por el Tribunal Superior del Distrito Judicial de Bogotá que sostiene que la acción impetrada -la acción de tutela- no es el mecanismo idóneo para la defensa de estos derechos, ya que el alcance de esta no abarca cuestiones de tal nivel de complejidad, como sí lo sería para una acción popular, que es un mecanismo constitucionalmente establecido para la defensa de derechos colectivos como el ambiente y que, además, para el caso concreto resultaba procedente.

Sin embargo, en esta problemática no se aborda únicamente la salvaguarda del derecho colectivo a gozar de un ambiente sano, sino que se estudia la vulneración de derechos fundamentales de los accionantes, algunos de los cuales son grupos en estado de vulnerabilidad -menores de edad-. Surge por tanto la inquietud de si la acción popular tiene el alcance para salvaguardar estos intereses jurídicos.

Para resolver tal situación, tras estudiar el fallo impugnado la Corte Suprema de Justicia decide acoger varios criterios, de orden doctrinal, normativo y jurisprudencial; recogidos en gran parte en la sentencia SU-1116 de 2001 de la Corte Constitucional, según la cual, si bien es cierto que en principio la acción de tutela tiene como finalidad la protección de derechos fundamentales 
individuales, excepcionalmente puede perseguirse la protección de intereses colectivos como el ambiente siempre que se cumplan los siguientes requisitos:

(i.) Que exista una conexidad entre la vulneración de derechos colectivos y la violación de derechos fundamentales, de tal forma que la amenaza de estos sea consecuencia directa e inmediata de la transgresión de aquellos; (ii.) que quién o quienes presenten la acción de tutela deben ser los titulares del derecho fundamental lesionado, dado el carácter subjetivo de los derechos fundamentales y de la tutela; (iii.) La violación a los derechos fundamentales debe ser palpable, encontrarse probada dentro del expediente, no puede ser hipotética. (Corte Constitucional, sentencia SU-1116 de 2001)

En la citada sentencia de unificación se sostiene que lo que realmente se busca en este tipo de casos es el amparo de los derechos fundamentales, aun cuando con la orden judicial se derive el resguardo o restablecimiento de derechos colectivos en situación de potencial vulnerabilidad.

La Corte Suprema concluyó que existe un peligro de daño en la medida que se encuentra acreditado que según los reportes del Ideam las emisiones de gases efecto invernadero provocadas por la deforestación generan una elevación en la temperatura de Colombia que aumentará gradualmente hasta el año 2070 donde alcanzará un incremento de $1,7^{\circ}$ centígrados (sentencia ST4360 de 2018).

En cuanto al principio de la equidad intergeneracional, íntimamente ligado con el concepto de desarrollo sostenible, la Corte acogió los postulados de los accionantes en la acción de tutela: debe existir una comprensión no solo entre las generaciones presentes y las generaciones futuras que aún no existen, sino entre quienes hoy toman las decisiones y quienes enfrentarán las consecuencias de ellas más adelante (Rodríguez et al., 2018). En el caso concreto la Corte consideró que son las futuras generaciones quienes tendrán que afrontar las derivaciones del aumento en las temperaturas.

Además, decide seguir la Corte con la visión biocéntrica adoptada por la Corte Constitucional en Sentencia T-622 de 2016, en la cual se reviste a la naturaleza como un pleno sujeto de derechos respecto al hombre, quien no se encuentra en una posición hegemónica ni preponderante sobre ella, sino que hace parte de la misma.

Otro argumento que plantea la Corte Suprema de Justicia -remitiéndose a la jurisprudencia de la Corte Constitucional- son las similitudes que presentan las acciones de tutela y popular, en tanto ambas buscan la protección de intereses jurídicos de primer nivel. En el primer caso de orden individual y en el segundo de orden colectivo; ambos en una potencial situación de amenaza por la acción u omisión de autoridades públicas o de particulares.

En últimas, lo que se busca es prevenir un perjuicio irremediable en tanto que la acción de tutela es viable como mecanismo transitorio para evitar la ocurrencia de un perjuicio, aunque existan otros mecanismos para la defensa del derecho, la acción popular no está supeditada a la ocurrencia del daño del bien colectivo, sino que es suficiente con que se amenace o haya peligro de daño. De esta manera, cuando hay una relación estrecha entre derechos colectivos y derechos fundamentales es procedente la acción de tutela por la imposibilidad de separar ambas clases de derechos (sentencia ST4360 de 2018). 
Fueron estos los parámetros tomados en cuenta por la Corte Suprema de Justica para determinar que los accionantes buscaban la protección de sus intereses subjetivos, los cuales se veían amenazados o resultaban negativamente afectados por la creciente deforestación del Amazonas y el consecuente cambio en las temperaturas del país, estando en peligro inminente los ecosistemas de este territorio.

Así, este alto tribunal decide revocar la decisión del a quo amparando los derechos esbozados por los jóvenes, configurando a favor de la Amazonía colombiana el estatus de 'sujeto de derechos'. Este reconocimiento se dio de manera posterior a la sentencia T-622 de 2016 de la Corte Constitucional en la que se reconoció al río Atrato como un ente sujeto de derechos, y fue a su vez un precedente clave para que el Páramo de Pisba fuese reconocido en igual sentido por el Tribunal Administrativo de Boyacá.

Con la sentencia, ordena la creación de un plan que pueda ejecutarse a corto, mediano y largo plazo, que al mismo tiempo que adopte medidas para disminuir la tasa de deforestación haga frente a los efectos del cambio climático. Ordena la participación de los actores secundarios y principales del conflicto e instruye para la construcción de un Pacto Intergeneracional por la vida del Amazonas colombiano, esto con el propósito de reducir a cero la deforestación y las emisiones de gases de efecto invernadero (sentencia ST4360 de 2018).

Decretó en ese mismo sentido la actualización e implementación de los planes de ordenamiento territorial de todos los municipios de la Amazonía colombiana afectados por esta problemática, finalmente, estableció para las corporaciones autónomas, Coorpoamazonía, CDA y Cormacarena la realización de un plan de acción que contrarreste con medidas policivas, judiciales o administrativas los problemas ocasionados por la deforestación.

Con el propósito de que la sentencia emitida no se quedará solo en el papel sino que fuera una realidad llevada a la práctica, el 15 de mayo del año en curso, los jóvenes accionantes junto a Dejusticia se reunieron con miembros de las autoridades del Ministerio de medio ambiente, algunos académicos y parte de la sociedad civil con el fin de planificar detalladamente las estrategias y medidas que se tomarán para ejecutar el Pacto Intergeneracional por la vida de la Amazonía (Pivac), (sentencia ST4360 de 2018).

Para la elaboración de este pacto no solo se ha requerido la participación de los entes involucrados, accionantes y accionados, sino que además se le ha dado la posibilidad a la comunidad en general de que contribuyan con sus propuestas e ideas, ya que el medio ambiente y la conservación de la Amazonía son intereses jurídicos que conciernen a todos (sentencia ST4360 de 2018).

\section{Conclusiones}

La importancia del instrumento para caracterizar conflictos ambientales diseñado por Rodríguez (2014) yace en el enfoque múltiple de impactos -sociales, ambientales y económicos- que se dimensionan sobre un caso con incidencia en lo ambiental. Esto le permite al investigador contar con una valiosa herramienta para abordar desde casi cualquier óptica diversas problemáticas derivadas del conflicto. 
Al aplicar este instrumento lo que se buscó fue caracterizar el conflicto ambiental a partir de la documentación aportada en la acción de tutela, en la postura de los actores principales y en los aspectos sociales, económicos y ambientales que les llevaron a solicitar la protección jurisdiccional de sus derechos vulnerados, en este caso, a través de un mecanismo que a priori no era el indicado para tales efectos.

Con todo, la Corte Suprema de Justicia al estudiar el caso contempló factores tales como el nexo causal entre cambio climático y deforestación amazónica, atendiendo a los principios que orientan las políticas ambientales, entre estos el principio de equidad intergeneracional, dado que serán las generaciones futuras quienes tendrán que enfrentar los factores de deterioro ambiental.

Asimismo, tuvo muy en cuenta el principio de sostenibilidad que propugna por la gestión eficiente de recursos por parte de las generaciones actuales para no comprometer los de las generaciones venideras, así como el principio de solidaridad y corresponsabilidad del Estado para contrarrestar la abrupta reducción boscosa.

Esto fue posible gracias a la normatividad, doctrina y jurisprudencia, con el propósito de analizar las implicaciones de reconocer a la Amazonía como sujeto de derechos, esto es, implementar en la práctica postulados del biocentrismo. Gracias a ello se dejaron sentadas las bases para un futuro seguimiento al Pacto Intergeneracional para la vida del Amazonas que se suscribió entre los actores principales del conflicto y el Estado.

Un último aspecto a tener en cuenta, a manera de ejercicio reflexivo, son los retos a los que se deben enfrentar las autoridades tras el reconocimiento por vía jurisprudencial de la naturaleza como sujeto de derechos. Deberá el Estado determinar si el ejercicio de las acciones tendientes a proteger este "nuevo" interés jurídico recaerá en todos los individuos o solo de algunas autoridades.

Igualmente, pese a ser un concepto novedoso y progresista, la aplicación de postulados del biocentrismo traerá consigo otros desafíos, tales como establecer cuáles son los derechos que la naturaleza posee en concreto, o en qué momento se invocaría su protección. En definitiva, el biocentrismo nos invita a replantear nuestra forma de relacionarnos con los demás seres vivos, además de ofrecer una visión innovadora del derecho ambiental.

\section{Referencias bibliográficas}

Armenteras, D., Rudas, G., Rodríguez, N., Sua, S., y Romero, M. (2006). Patterns and causes of deforestation in the Colombian Amazon. Ecological Indicators, 6(2), 353-368. doi.org/10.1016/j. ecolind.2005.03.014.

Arria, M., Rodríguez-Morales, A. J., y Franco-Paredes, C. (2005). Ecoepidemiología de las enfermedades tropicales en países de la cuenca amazónica. Revista Peruana de Medicina Experimental y Salud Publica, 22(3), 236-240.

Bernardes, C., y Riso, M. (2014). Generation of domestic solid waste in rural areas: case study of remote communities in the Brazilian Amazon. Human Ecology, 42(4), 617-623. doi.org/10.1007/ s10745-014-9679-z. 
Bunyard, P., y Herrera, F. (2012). El rol de la selva amazónica en la formación de las lluvias en Colombia. Intekhnia, 7(1), 27-36.

Cameron, J y Abouchar, J. (1991). The precautionary principle: a fundamental principle of law and policy for the protection of the global environment. Boston College International and Comparative Law Review, 14, 1.

Congreso de la República de Colombia. (22 de diciembre de 1993). Ley 99 de 1993. Diario Oficial No. 41.146. Recuperado de http://www.secretariasenado.gov.co/senado/basedoc/ ley_0099_1993.html

Congreso de la República de Colombia, 5 de agosto de 1998. Ley 472 de 1998. Diario Oficial No. 43.357 Recuperado de http://www.secretariasenado.gov.co/senado/basedoc/ley_0472_1998. html

Corte Constitucional, Sala Plena. (24 de octubre de 2001). Sentencia SU-1116 de 2001. [M.P. Eduardo Montealegre Lynett].

Corte Constitucional, Sala Sexta de Revisión. (10 de noviembre de 2016). Sentencia T-622 de 2016. [M.P. Jorge Iván Palacio Palacio]

Corte Suprema de Justicia, Sala de Casación de civil. (5 de abril del 2018). Sentencia STC4360 de 2018. [M.P. Luis Armando Tolosa Villabona].

Gudynas, E. (2009). La ecología política del giro biocéntrico en la nueva Constitución de Ecuador. Revista de Estudios Sociales, 32, 34-47. doi/pdf/10.7440/res32.2009.02

IDEAM (2017). Decimoprimer Boletín de Alertas Tempranas de Deforestación (AT-D), Segundo trimestre 2017. Recuperado de http://documentacion.ideam.gov.co/cgi-bin/koha/opac-imageviewer.pl?biblionumber $=38232$.

Mariné, A. (2007). El agua en la agricultura. Agricultura: Revista agropecuaria, 902, 876-877.

Ministerio de Ambiente y Desarrollo Sostenible (2017). Estrategia integral de control a la deforestación y gestión de los bosques [Documento de trabajo] Recuperado de http://www.minambiente.gov.co/images/EICDGB_1.0_AGOSTO_9_2017.pdf

Ministerio de Ambiente y Desarrollo Sostenible. (2018). Corporaciones Autónomas Regionales, Instrumentos de Planificación Regional. Recuperado dehttp://www.minambiente.gov.co/index. php/component/content/article/885-plantilla-areas-planeacion-y-seguimiento-33

Padilla, J. C., Lizarazo, F. E., Murillo, O. L., Mendigaña, F. A., Pachón, E., y Vera, M. J. (2017). Epidemiología de las principales enfermedades transmitidas por vectores en Colombia, 1990-2016. Biomédica, 37(2), 27-40. doi: 10.7705/biomedica.v34i2.3769

Pereira de Lima, R. (2001). La selva amazónica como problema geográfico. Documents d'Anàlisi Geogràfica, 38, 99-108. 
Pinzón-Pinto. A. (2010). Dinámica del agua en el suelo amazónico. En H. Bernal, C. Sierra, M. Onaindia y M. Angulo (Eds.) Amazonía y agua: desarrollo sostenible en el siglo XXI (pp. 73-81). Bilbao: Servicio Editorial de la Unesco Etxea.

PNUMA (2009). Perspectivas del Medio Ambiente en la Amazonía. Recuperado de http://www. pnuma.org/deat1/pdf/geoamazonia_spanish_FINAL.pdf

Rodríguez, G.A. (2014). Oportunidades y limitaciones de la consulta previa en la prevención y solución de conflictos ambientales en territorios indígenas en Colombia [Tesis doctoral]. Bogotá: Universidad Externado de Colombia

Rodríguez, G. A. y Páez, I. A. (2012). La consagración de los derechos ambientales en las constituciones políticas de Colombia, Ecuador y Bolivia. (pp. 1-53) En G. A. Rodríguez \& I. Páez (2012). Temas de Derecho Ambiental: una mirada desde lo público. Bogotá: Editorial Universidad del Rosario.

Rodríguez, J. et al (2018-01-28). Acción de tutela presentada ante el Tribunal Superior del Distrito Judicial de Bogotá - Sala Civil. Accionantes: José Daniel Rodríguez Peña y otros. Accionados: Presidencia de la República de Colombia y otros. Recuperado el 12 de diciembre de 2018 de: https://cdn.dejusticia.org/wp-content/uploads/2018/01/TutelaCambioClim\%C3\%A1tico.pdf

Salamanca, A. et al. (2018-02-16) Impugnación fallo de primera instancia. Radicado No. 11001 220300020180031900 Accionantes: Andrés Mauricio Salamanca Mancera y otros. Accionados: Presidencia de la República de Colombia y otros. Recuperado el 06 de marzo de 2019 de https://cdn.dejusticia.org/wp-content/uploads/2018/05/Impugnacio\%CC\%81n-Sentencia-1-Instancia-Demanda-Generaciones-Futuras-v.-Minambiente-16022018.pdf?x54537

Urrutia, R., y Vuille, M. (2009). Climate change projections for the tropical Andes using a regional climate model: Temperature and precipitation simulations for the end of the 21st century. Journal of Geophysical Research: Atmospheres, 114(D2). doi.org/10.1029/2008JD011021.

Veyrunes, E. (2008). Las amenazas percibidas para la Amazonía: un estado del arte en términos de seguridad ambiental. Bogotá: Editorial Universidad del Rosario.

Viviroli, D., Archer, D. R., Buytaert, W., Fowler, H. J., Greenwood, G., Hamlet, A. F., ... \& Lorentz, S. (2011). Climate change and mountain water resources: overview and recommendations for research, management and policy. Hydrology and Earth System Sciences, 15(2), 471-504. doi. org/10.5194/hess-15-471-2011 